\title{
Regulatory Submission Unit Type
}

National Cancer Institute

\section{Source}

National Cancer Institute. Regulatory Submission Unit Type. NCI Thesaurus. Code C70859.

A particular categ ory of a regulatory submission regarded as having particular characteristics, purposes, and causing different regulatory requests. 